\title{
UK Renal Registry 14th Annual Report: Appendix H Coding: Ethnicity, EDTA Primary Renal Diagnoses, EDTA Causes of Death
}

\section{H1: Ethnicity coding}

Ethnicity data is recorded in the clinical information systems in the individual renal centres in the format of $9 S . .$. read codes. If extracted from local PAS systems in a different format, it is recoded to the 9S... format by the centre, before being sent to the UKRR. For report analyses, ethnic categories are condensed into five groups (White, Asian, Black, Chinese and other). For some analyses Chinese are grouped into other.

\begin{tabular}{|c|c|c|c|c|}
\hline Read code & Ethnic category & Assigned group & Old PAS & New PAS \\
\hline 9S1.. & White & White & 0 & A1 \\
\hline 9SA9. & Irish (NMO) & White & & B1 \\
\hline 9SAA. & Greek Cypriot (NMO) & White & & CG \\
\hline 9SAB. & Turkish Cypriot (NMO) & White & & CJ \\
\hline 9SAC. & Other European (NMO) & White & & $\mathrm{C} 1$ \\
\hline 9S6.. & Indian & Asian & 4 & H1 \\
\hline 9S7.. & Pakistani & Asian & 5 & J1 \\
\hline 9S8.. & Bangladeshi & Asian & 6 & K1 \\
\hline 9SA6. & East African Asian & Asian & & \\
\hline 9SA7. & Indian Subcontinent & Asian & & \\
\hline 9SA8. & Other Asian & Asian & & L1 \\
\hline 9S2.. & Black Caribbean & Black & 1 & M1 \\
\hline 9S3.. & Black African & Black & 2 & N1 \\
\hline 9S4.. & Black/Other/non-mixed origin & Black & 3 & P1 \\
\hline 9S41. & Black British & Black & & $\mathrm{PD}$ \\
\hline $9 S 42$. & Black Caribbean & Black & & \\
\hline $9 S 43$. & Black North African & Black & & \\
\hline $9 S 44$. & Black other African country & Black & & \\
\hline $9 S 45$. & Black East African Asian & Black & & \\
\hline $9 S 46$. & Black Indian subcontinent & Black & & \\
\hline $9 S 47$. & Black Other Asian & Black & & \\
\hline $9 S 48$. & Black Black Other & Black & & $\mathrm{PE}$ \\
\hline 9S5.. & Black other/mixed & Black & & \\
\hline $9 \mathrm{~S} 51$. & Other Black - Black/White origin & Black & & GC \\
\hline $9 \mathrm{~S} 52$. & Other Black - Black/Asian origin & Black & & GA \\
\hline 9S9.. & Chinese & Chinese & 7 & R1 \\
\hline 9T1C. & Chinese & Chinese & & \\
\hline 9SA.. & Other ethnic non-mixed (NMO) & Other & & \\
\hline 9SA1. & British ethnic minority specified (NMO) & Other & & \\
\hline 9SA2. & British ethnic minority unspecified (NMO) & Other & & \\
\hline 9SA3. & Caribbean Island (NMO) & Other & & \\
\hline
\end{tabular}

\section{KARGER}

Fax +41 613061234

E-Mail karger@karger.ch

www.karger.com
(C) 2012 S. Karger AG, Basel

$1160-2110 / 12 / 1185-0309 \$ 38.00 / 0$

Accessible online at: www.karger.com/nec
UK Renal Registry, Southmead Hospital, Southmead Road, Bristol, BS10 5NB, UK

Email: renalregistry@renalregistry.nhs.uk 


\begin{tabular}{|c|c|c|c|c|}
\hline Read code & Ethnic category & Assigned group & Old PAS & New PAS \\
\hline 9SA4. & North African Arab (NMO) & Other & & \\
\hline 9SA5. & Other African countries (NMO) & Other & & \\
\hline 9SAD. & Other ethnic NEC (NMO) & Other & & S1 \\
\hline 9SB.. & Other ethnic/mixed origin & Other & 8 & \\
\hline 9SB1. & Other ethnic/Black/White origin & Other & & E1 \\
\hline 9SB2. & Other ethnic/Asian/White origin & Other & & F1 \\
\hline 9SB3. & Other ethnic/mixed White origin & Other & & \\
\hline 9SB4. & Other ethnic/Other mixed origin & Other & & G1 \\
\hline
\end{tabular}

H2: EDTA primary renal diagnoses

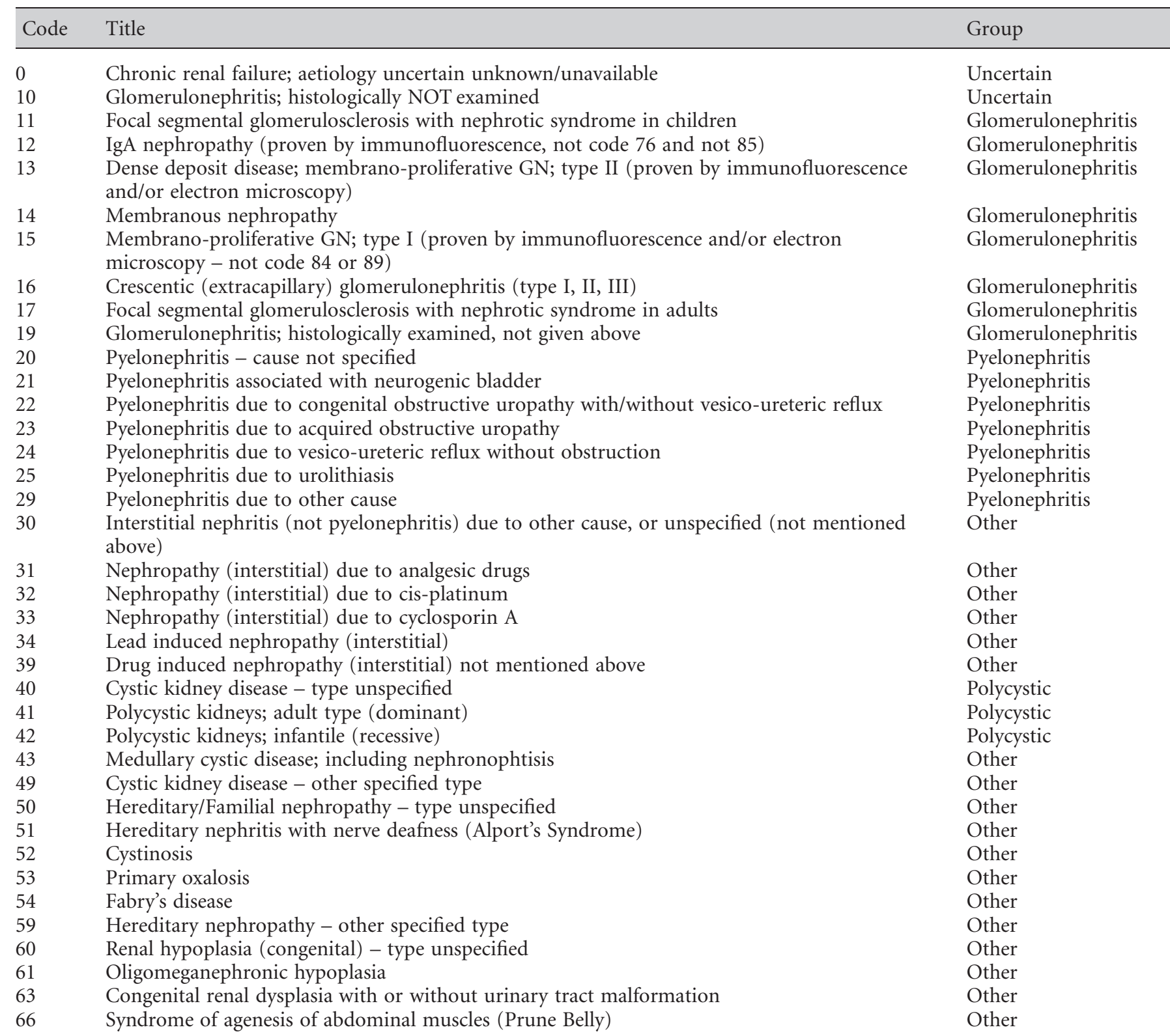




\begin{tabular}{lll}
\hline Code & Title & Group \\
\hline 70 & Renal vascular disease - type unspecified & Renal vascular disease \\
71 & Renal vascular disease due to malignant hypertension & Hypertension \\
72 & Renal vascular disease due to hypertension & Hypertension \\
73 & Renal vascular disease due to polyarteritis & Renal vascular disease \\
74 & Wegener's granulomatosis & Other \\
75 & Ischaemic renal disease/cholesterol embolism & Renal vascular disease \\
76 & Glomerulonephritis related to liver cirrhosis & Other \\
78 & Cryoglobulinemic glomerulonephritis & Other \\
79 & Renal vascular disease - due to other cause (not given above and not code 84-88) & Renal vascular disease \\
80 & Type 1 diabetes with diabetic nephropathy & Diabetes \\
81 & Type 2 diabetes with diabetic nephropathy & Diabetes \\
82 & Myelomatosis/light chain deposit disease & Other \\
83 & Amyloid & Other \\
84 & Lupus erythematosus & Other \\
85 & Henoch-Schoenlein purpura & Other \\
86 & Goodpasture's Syndrome & Other \\
87 & Systemic sclerosis (scleroderma) & Other \\
88 & Haemolytic Ureaemic Syndrome (including Moschcowitz Syndrome) & Other \\
89 & Multi-system disease - other (not mentioned above) & Other \\
90 & Tubular necrosis (irreversible) or cortical necrosis (different from 88) & Other \\
91 & Tuberculosis & Other \\
92 & Gout nephropathy (urate) & Other \\
93 & Nephrocalcinosis and hypercalcaemic nephropathy & Other \\
94 & Balkan nephropathy & Other \\
95 & Kidney tumour & Other \\
96 & Traumatic or surgical loss of kidney & Other \\
98 & Not known & Missing \\
99 & Other identified renal disorders & Other \\
199 & Code not sent & Missing \\
& &
\end{tabular}




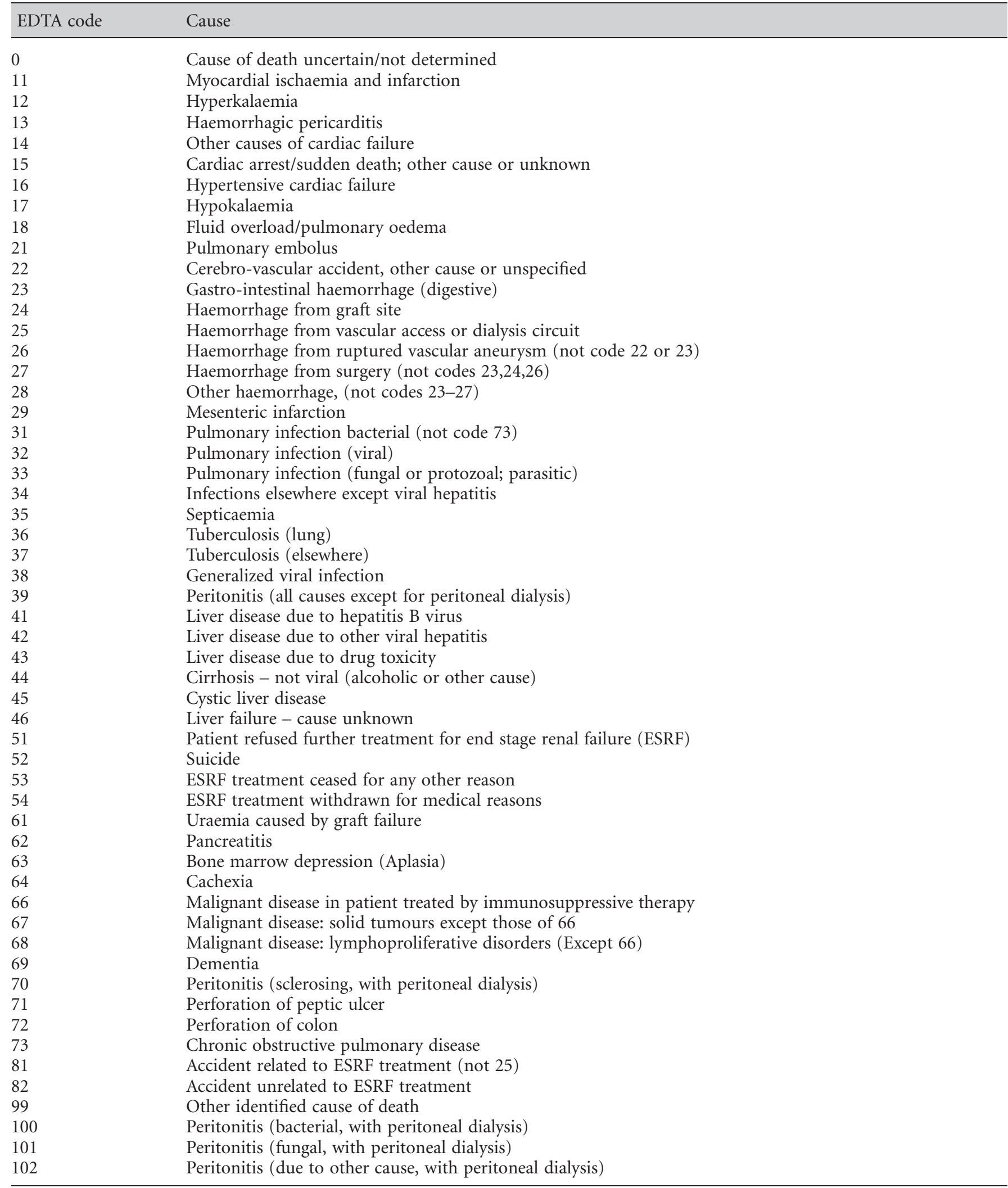

